\title{
The human circulating miRNome reflects multiple organ disease risks in association with short-term exposure to traffic-related air pollution
}

\author{
Julian Krauskopf $^{\mathrm{a}, *}$, Florian Caiment ${ }^{\mathrm{a}}$, Karin van Veldhoven ${ }^{\mathrm{b}}$, Marc Chadeau-Hyam ${ }^{\mathrm{b}}$, \\ Rudy Sinharay ${ }^{\mathrm{c}}$, Kian Fan Chung ${ }^{\mathrm{c}}$, Paul Cullinan ${ }^{\mathrm{c}}$, Peter Collins ${ }^{\mathrm{c}}$, Benjamin Barratt ${ }^{\mathrm{d}}$, \\ Frank J. Kelly ${ }^{\mathrm{d}}$, Roel Vermeulen ${ }^{\mathrm{e}}$, Paolo Vineis ${ }^{\mathrm{b}}$, Theo M. de Kok ${ }^{\mathrm{a}}$, Jos C. Kleinjans ${ }^{\mathrm{a}}$ \\ a Department of Toxicogenomics, GROW School for Oncology and Developmental Biology, Maastricht University, Maastricht, The Netherlands \\ ${ }^{\mathrm{b}}$ MRC-PHE Centre for Environment and Health, Department of Epidemiology and Biostatistics, School of Public Health, Imperial College, London, UK \\ ${ }^{\mathrm{c}}$ National Heart and Lung Institute, Imperial College London, UK \\ ${ }^{\mathrm{d}}$ MRC-PHE Centre for Environment and Health, Analytical \& Environmental Sciences, King's College London, UK \\ e Institute for Risk Assessment Sciences, Utrecht University, Utrecht, The Netherlands
}

\section{A R T I C L E I N F O}

Editor: Kalantzi Olga Ioanna

Keywords:

Air pollution

Diesel exhaust

Extracellular microRNAs

Biomarkers

Health risk assessment

Liquid biopsies

\begin{abstract}
A B S T R A C T
Traffic-related air pollution is a complex mixture of particulate matter (PM) and gaseous pollutants, such as nitrogen dioxide (NO2). PM exposure contributes to the pathogenesis of many diseases including several types of cancer, as well as pulmonary, cardiovascular and neurodegenerative diseases. Also exposure to NO2 has been related to increased cardiovascular mortality. In search of an early diagnostic biomarker for improved air pollution-associated health risk assessment, recent human studies have shown that certain circulating miRNAs are altered upon exposure to traffic-related air pollutants. Here, we present for the first time a global analysis of the circulating miRNA genome in an experimental cross-over study of a human population exposed to traffic-related air pollution. By utilizing next-generation sequencing technology and detailed real-time exposure measurements we identified 54 circulating miRNAs to be dose- and pollutant species-dependently associated with PM10, PM2.5, black carbon, ultrafine particles and NO2 already after $2 \mathrm{~h}$ of exposure. Bioinformatics analysis suggests that these circulating miRNAs actually reflect the adverse consequences of traffic pollution-induced toxicity in target tissues including the lung, heart, kidney and brain. This study shows the strong potential of circulating miRNAs as novel biomarkers for environmental health risk assessment.
\end{abstract}

\section{Introduction}

Numerous epidemiological studies have associated exposure to traffic-related air pollution (TRAP) with increased risk of cardiovascular disease, (Brook et al., 2010; Lee et al., 2014) respiratory disease, (Xing et al., 2016) several types of cancer, including lung and breast cancer, (Hamra et al., 2014; Tagliabue et al., 2016) and more recently also of neurodegenerative diseases (Cacciottolo et al., 2017; Chen et al., 2017) and kidney disease (Bowe et al., 2017). A number of gaseous pollutants such as nitrogen dioxide (NO2) as well as particulate matter components (PM) are routinely monitored to characterize TRAP exposure. PM is a complex mixture of fine particles with a diameter of $10 \mu \mathrm{m}$ or less (PM10), a diameter of $2.5 \mu \mathrm{m}$ or less (PM2.5), black carbon (BC), ultrafine particles with a diameter of $0.1 \mu \mathrm{m}$ or less (UFP) and soot (Falcon-Rodriguez et al., 2016). Upon inhalation PM penetrates deeply into the lungs from where, depending on their size, particles are capable of entering the circulation and being distributed to distal organs such as the heart, spleen or liver (Falcon-Rodriguez et al., 2016; Li et al., 2015; Yaghjyan et al., 2017). It has even been reported that ultrafine PM crosses the blood-brain barrier and translocates from the circulation to the brain (Oberdorster et al., 2004). Further, it has been demonstrated that PM triggers oxidative stress in the respiratory tract and that this might induce a systemic inflammatory cascade, thus increasing the risk for respiratory and cardiovascular diseases (Bollati et al., 2015). Presumably, pollutants, once distributed over the whole body, may cause a similar cascade of oxidative stress and inflammation in target organs, thereby increasing risks for cancer and neurodegenerative disease (Block \& Calderon-Garciduenas, 2009). However, the precise molecular mechanisms that link TRAP exposure to increased disease risks are still poorly understood which hampers the development of dedicated biomarkers capable of informing on relevant molecular mechanisms of action.

\footnotetext{
* Corresponding author.

E-mail address: j.krauskopf@maastrichtuniversity.nl (J. Krauskopf).
} 
Several studies have thus highlighted the impact of environmental exposure on gene expression profiles, (van Leeuwen et al., 2006) DNAmethylation patterns, (Georgiadis et al., 2016) and p53 status (Intarasunanont et al., 2012). More recently, environmental exposureinduced alterations in microRNA (miRNA) levels have been described (Krauskopf et al., 2017a). These small non-coding RNAs are involved in the posttranscriptional regulation of gene expression, and consequently are involved in virtually all cellular processes (Bartel, 2009). Furthermore, while these fine-tuners of gene expression are capable of adjusting to internal and external conditions, they also exhibit tissue/ organ specific expression patterns (Landgraf et al., 2007). As a consequence of organ injury, cells may leak their content including the highly stable protein-bound miRNAs, into the peripheral circulation (Turchinovich et al., 2012). Given the fact that certain miRNAs are more abundantly expressed in specific organs, circulating miRNA (cmiRNA) signatures may thus also reflect organ-specific responses to exposure (Krauskopf et al., 2015). Furthermore, through active secretion, extracellular vesicle-bound cmiRNAs may act as mediators in intercellular and interorgan communication (Hunter et al., 2008). Therefore, cmiRNAs leaked or released from organs into the circulation, have become a new promising class of biomarkers capable of non-invasively interrogating organ pathogenesis and organ-toxic mechanisms from so called 'liquid biopsies' (Krauskopf et al., 2015).

To date, most reported air pollution-induced changes in miRNA expression have been identified in solid tissues in animal models (Vrijens et al., 2015). The first evidence on PM exposure-related modifications in cmiRNA levels in humans was provided through investigating healthy steel plant workers. This study identified 2 vesicleassociated miRNAs that were elevated after occupational exposure to metal-rich PM (Bollati et al., 2015). Additionally, a study on long term exposure to ambient air pollution (6 month or 1 year) identified the elevation of 5 vesicle-associated cmiRNAs in the serum of healthy subjects (Rodosthenous et al., 2016). Another study among children identified 2 cmiRNAs in the extracellular fraction of saliva to be significantly altered with long-term ultrafine PM exposure (Vriens et al., 2016).

These studies provided evidence that the extracellular miRNA genome (miRNome) is affected by TRAP exposure through utilizing targeted approaches, and were consequently restricted to analyzing a priori known air pollution-associated miRNAs. In the current study, we present for the first time a global analysis of the circulating miRNome by applying next generation sequencing technology and real-time exposure measurements in an experimental cross-over study of human volunteers $(n=24)$ following short-term traffic-related air pollution exposure. This study demonstrates the potential of circulating miRNAs as novel biomarkers for health risk assessment in relation to environmental exposure-induced target tissue pathogenesis.

\section{Methods}

\subsection{Selection of the population}

Plasma samples were collected during a randomized experimental crossover study in which non-smoking participants, either healthy or suffering from ischemic heart disease (IHD) or chronic obstructive pulmonary disease (COPD), walked for $2 \mathrm{~h}$ along Oxford Street in London (where only diesel-powered buses and taxicabs are permitted). In a separate session the same subjects also walked for $2 \mathrm{~h}$ through traffic-free Hyde Park. In order to balance between sufficient exposure and what is acceptable for the participants we decided for a $2 \mathrm{~h}$ exposure window which also has been applied successfully in an earlier study (McCreanor et al., 2007). Sessions were segregated by a period of $>3$ weeks. Participants walked about $6 \mathrm{~km}$ during each exposure period, at a steady pace on predefined paths, resting for $15 \mathrm{~min}$ every half hour. During each exposure session, personal ambient air pollution levels of PM were assessed using a real-time condensation particle
Table 1

Study population data.

\begin{tabular}{lllllll}
\hline & \# Subjects & $\begin{array}{l}\text { Age, } \\
\text { mean } \\
\text { (SD) }\end{array}$ & $\begin{array}{l}\text { BMI, } \\
\text { mean } \\
\text { (SD) }\end{array}$ & \# Healthy & \# COPD \# IHD \\
\hline All subjects & 24 & $65.1(7.7)$ & $25(4.8)$ & 8 & 8 & 8 \\
Male & 12 & $65(10)$ & $24.9(3.5)$ & 3 & 4 & 5 \\
Female & 12 & $64.9(5.1)$ & $25.1(6)$ & 5 & 4 & 3 \\
\hline
\end{tabular}

counter (Model 3007, TSI). Particles smaller than $2.5 \mu \mathrm{m}$ in aerodynamic diameter were collected on an air sampler. BC was measured according to the National Institute for Occupational Safety and Health guidelines (Method 5040, Sunset Laboratory). Using a sampling pump, NO2 was collected on C18 Sep-Pak cartridges coated with potassium hydroxide and triethanolamine and analyzed using ion chromatography (McCreanor et al., 2007). Our comprehensive monitoring design aimed to account for each of the major ambient air pollution factors that differed between sites. To the best of our knowledge there are no major differences with respect to any other factors not taken into account. In total 119 subjects completed the exposure assessment (Sinharay et al., 2017). For the present study, we selected 24 non-smoking participants, based on the maximal change in PM exposure between Hyde Park and Oxford Street while balancing for gender (Table 1). The study was approved by the UK National Research Ethics Service (London City Road and Hampstead Ethics Committee; Research Ethics Number 12/ LO/1064, 01/08/2012). All participants gave written informed consent. The studies were conducted in accordance with the approved guidelines and regulations.

\subsection{Analytical procedures}

After each exposure session the participants were transferred to the Royal Brompton Hospital where blood samples were collected. Plasma samples were recovered from plasma separator tubes following centrifugation of whole blood at $1600 \mathrm{~g}$ for $10 \mathrm{~min}$ at $4{ }^{\circ} \mathrm{C}$. To avoid contamination of the plasma cmiRNAs with blood cell-derived miRNAs, the plasma was subjected to a second centrifugation step at $16,000 \mathrm{~g}$ before being stored at $-80^{\circ} \mathrm{C}$ (Kroh et al., 2010). Plasma cmiRNAs were isolated using the miRNeasy Serum/Plasma kit (Qiagen) and the quality was evaluated on a Bioanalyzer using the small RNA Kit (Agilent). Samples were included in the analysis when showing a minimum of $1 \mathrm{ng}$ RNA yield and a peak at 21 nucleotides. The sequencing libraries were prepared using the TruSeq Small RNA-Seq Preparation Kit (Illumina), and sequenced by a HiSeq 2500 (Illumina) (Krauskopf et al., 2017b) according to the manufacture's protocol (GEO accession: GSE106221).

\subsection{Statistical data analysis}

The quality of the sequencing data was assessed by FastQC and subsequently processed using the miRge pipeline and miRBase (release 21) (Baras et al., 2015; Griffiths-Jones et al., 2006). In R, we set a threshold for a cmiRNA to be detected with a minimum of 100 raw sequencing reads across all samples. The raw data were quantile normalized and transformed to $\log 2$ counts per million ( $\log C P M)$ using the 'voom' function of the package 'limma' (Ritchie et al., 2015). Technical confounders (inter-day variations in library preparation and isolation batch effects) were adjusted for by applying a linear mixed model approach using the R package 'lme4' (Bates et al., 2015). After de-noising the data we used a multivariate normal model to find associations between the air pollutant exposure and cmiRNA using the R package 'nlme' (Pinheiro et al., 2014). For each exposure, an independent analysis was performed thereby also adjusting for sex, age, BMI and disease state (healthy, IHD or COPD). Walks were carried out Monday to 
Thursday. No attempt was made to match the day of the week when subjects returned for the second session as this would have been too restrictive to the subjects. However, the 'traffic exposure' site (Oxford Street) was only open to buses and taxis, with diesel buses representing the dominant emission source. As bus flows were consistent across all study days, day of the week was not regarded a significant confounder. Resulting $p$-values were controlled by the False Discovery Rate (FDR) at 5\% (Benjamini \& Hochberg, 1995).

Potential gene targets were derived from the experimentally validated miRNA-target interactions database miRTarBase (release 6) by only considering strong miRNA-gene interactions that have been validated by reporter assay, western blot or qPCR. Overrepresentation analysis of identified KEGG pathways retrieved from the potential gene targets was performed in ConsensusPathDB (release 32), using the gene targets for all detected cmiRNAs as a background list. Connections between pathways and diseases were retrieved from the KEGG DISEASE Database (updated: September 16, 2016).

To identify potential organs of origin of the detected cmiRNAs, we examined miRNA tissue enrichment using publicly available small RNA-seq data sets. The data were obtained from the Human miRNA Expression Database (Panwar et al., 2017) as well as from the sequence read archive (SRA012516) (Faghihi et al., 2010). The final dataset comprised 101 small RNA sequencing samples across 8 organs known to accumulate inhaled fine PM (Falcon-Rodriguez et al., 2016; Li et al., 2015; Yaghjyan et al., 2017). We applied a tissue specificity index (TSI), as described originally for mRNAs by Yanai et al., for each of the significant air pollutant exposure-associated cmiRNAs. The index is defined as:

$\mathrm{TSI}=\frac{\sum_{i}^{N}\left(1-x_{i}\right)}{N-1}$

Where $\mathrm{N}$ is the number of organs included in the analysis and $\mathrm{x}_{\mathrm{i}}$ is the expression of a miRNA, normalized by the value of the highest expressed miRNA (Yanai et al., 2005). A TSI $<0.5$ indicates that a miRNA is expressed across all the tissues whereas a higher TSI indicates that a miRNA is enriched in one or a few tissues.

\section{Results}

\subsection{Exposure range}

The subjects analyzed for cmiRNAs were exposed to a mean ambient air NO2 level of 7.9 (CI 5.9-9.8) $\mu \mathrm{g} / \mathrm{m}^{3}$ in Hyde Park and 18.1 (CI 15.1-21.1) $\mu \mathrm{g} / \mathrm{m}^{3}$ in Oxford Street. For PM2.5 the mean exposure level in Hyde Park was 5.6 (CI 4.5-6.8) $\mu \mathrm{g} / \mathrm{m}^{3}$ and 25.6 (CI 21-30.2) $\mu \mathrm{g} / \mathrm{m}^{3}$ in Oxford Street. For BC the exposure level was 1.0 (CI $0.8-1.3$ ) $\mu \mathrm{g} / \mathrm{m}^{3}$ in Hyde Park and 11.4 (CI 9.9-12.8) $\mu \mathrm{g} / \mathrm{m}^{3}$ in Oxford Street and for PM10 16.0 (CI 12.5-19.5) $\mu \mathrm{g} / \mathrm{m}^{3}$ in Hyde Park and 37.0 (CI 32.2-41.7) $\mu \mathrm{g} / \mathrm{m}^{3}$ in Oxford Street. For UFP the mean particle count was 5975 (CI 4815.4-7133.8) thousands $/ \mathrm{cm}^{3}$ in Hyde Park and 28,656 (CI 25803-31,509) thousands $/ \mathrm{cm}^{3}$ in Oxford Street. The exposure level densities for UFP, PM2.5, BC, PM10 and NO2 are presented in Fig. 1.

\subsection{Small RNA-sequencing}

The sequencing of the 48 samples from 24 subjects yielded a mean of 5.6 million ( \pm 2.8 million) high quality, preprocessed reads per sample of which on average 3.8 million ( \pm 2.1 million) could be assigned to the miRNA sequences from miRBase (release 21). Principal component analysis revealed that 2 outliers were present in the data. As a consequence these 2 subjects, as well as 2 further subjects with missing exposure measurements, were excluded from the analysis. The excluded subjects were not separated by any specific characteristics used in the analysis (Supplementary Table S1). In total, we identified 595 cmiRNAs across all plasma samples. The most abundant plasma
cmiRNA was miR-486-5p with $19.4 \log \mathrm{CPM}$, followed by miR-92a (16.6 $\log \mathrm{CPM})$, and miR-22-3p (14.4 $\log \mathrm{CPM})$. The upper quantile of all identified cmiRNAs ranged from $4.5 \log \mathrm{CPM}$ to $19.4 \log \mathrm{CPM}$, whereas the lower quantile ranged from -3.5 to $-1.4 \log \mathrm{CPM}$. The majority of the cmiRNAs present in the plasma varied between 4.5 and -3.5 $\log \mathrm{CPM}$.

\subsection{TRAP-associated cmiRNAs}

Using the multivariate normal model in combination with the linear mixed model for correction of technical random effects, we identified cmiRNAs significantly associated with pollutant-specific exposure levels (Fig. 2). We observed 6 cmiRNAs to be significantly associated with the NO2 levels, of which 4 were negatively and 2 positively correlated with exposure. Further, we identified 7 cmiRNAs, 5 negatively and 2 positively, associated with UFP counts. Analysis of PM2.5 levels resulted in 23 cmiRNAs, of which 12 were negatively and 11 positively associated with PM2.5 levels. We observed 26 cmiRNAs to be associated with BC levels of which 14 were negatively and 12 positively associated with exposure. For PM10 levels we identified $28 \mathrm{cmiRNAs}$ of which 17 were negatively and 11 positively associated with PM10 levels. Together, the traffic-related air pollutants (NO2, UFP, PM2.5, BC and PM10) were associated to 54 unique cmiRNAs (Supplementary Table S2). Fig. 3 presents the different plasma levels of those 9 cmiRNAs that were declared significant based on the more stringent Bonferroni correction (ensuring a family-wise error rate $<0.05$ ). When comparing the read outs after exposure in Hyde Park and upon exposure in Oxford Street, we observed decreased miR-133a-3p levels in 95\% of the subjects, in 85\% decreased miR-193b-3p levels, in 80\% increased miR1224-5p levels, in 85\% decreased miR-433-3p levels, in 80\% decreased miR-145-5p levels, in 65\% decreased miR-27a-5p levels, in $60 \%$ decreased miR-580-3p levels, in 55\% increased miR-3127-5p levels and in $75 \%$ decreased miR-6716-3p levels. This pair-wise comparison clearly demonstrates the consistency in cmiRNA response to ambient air pollutant exposure among the subjects. We did not detect any significant results upon stratification by disease state (COPD or IHD vs Healthy) or gender due to the limited number of samples.

\subsection{Origin of circulating miRNAs}

To assess the tissue origin of the detected cmiRNAs, we examined miRNA tissue enrichment using publicly available small RNA-seq data sets. From the 54 TRAP-affected cmiRNAs, we identified 7 to be highly expressed ( $\log 2 \mathrm{CPM}>10)$ in the lung, 8 in the breast, 3 in the heart, 6 in the kidney, 10 in the liver, 8 in the pancreas, 7 in the spleen and 7 in the brain.

Of the cmiRNAs significantly associated with air pollutant exposure, only 1 (miR-193b-3p) overlapped with the list of miRNAs associated with hemolysis, indicating that the potential plasma contamination with miRNAs from red blood cells was minimal (Kirschner et al., 2013).

By applying a tissue specificity index (TSI) we found that the cmiRNAs that were most abundant in the plasma, notably miR-25-3p, miR-30d-5p, let-7i-5p, miR-107 (plasma level > $5 \log 2 \mathrm{CPM}$ ), showed a low TSI and were consequently highly abundantly expressed across all target tissues. By contrast, low abundant cmiRNAs (plasma level $<5$ $\log$ CPM) showed a high TSI and appeared to be enriched in only one or just a few tissues. The miRNAs miR-133a-3p and miR-499a-5p, both enriched in the heart with a TSI of 0.60 and 0.64 , were present in the plasma at a mean of 3.7 and $-2.1 \log \mathrm{CPM}$, respectively. The brainenriched miR-433-3p showed a TSI of 0.58 with a plasma level of 0.7 $\log \mathrm{CPM}$, while the brain- and pancreas-enriched miR-1224-5p showed a TSI of 0.68 and was detected in the plasma at $3 \log C P M$. We identified the kidney-enriched miRNAs miR-3678-3p, miR-6741-3p, miR-68655p, miR-4687-5p, hsa-miR-627-3p and miR-636, all with a TSI $>0.68$ and plasma levels $<3.8 \log$ CPM. Furthermore, we identified miR-6514$3 p$ to be enriched in the breast, with a TSI of 1 and a plasma level of 
NO2

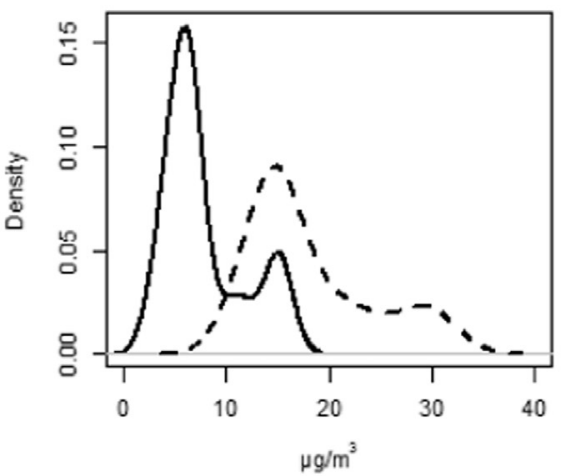

$\mathrm{BC}$

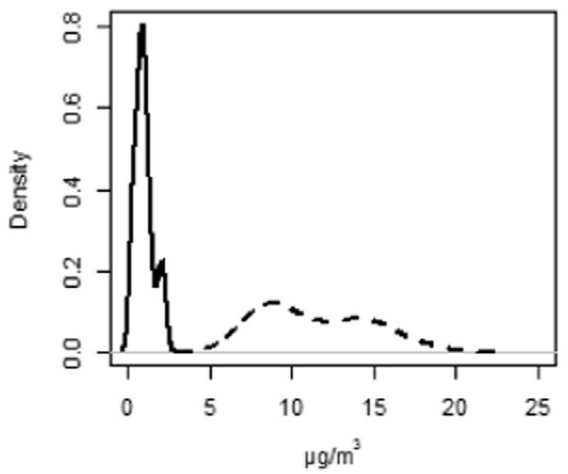

UFP

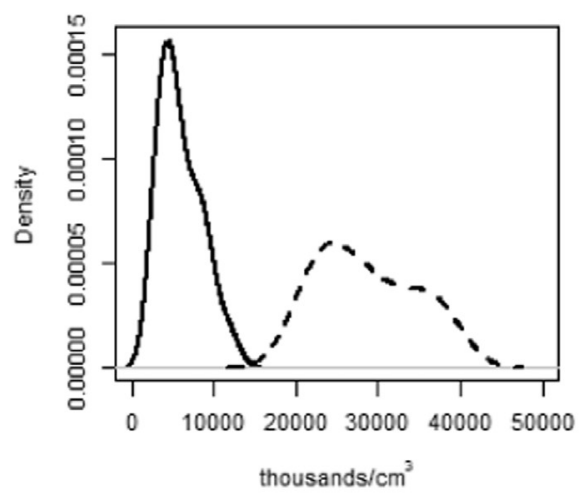

PM10

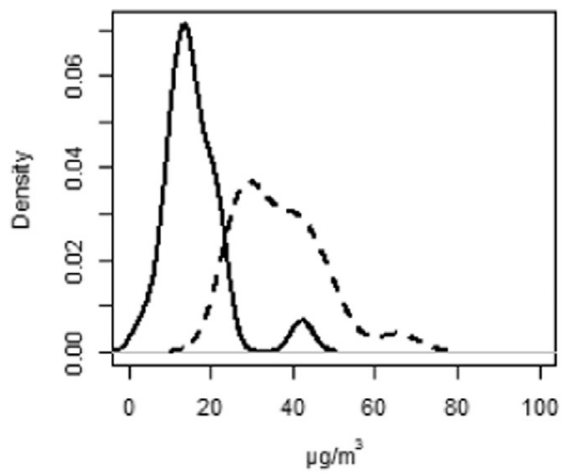

PM2.5
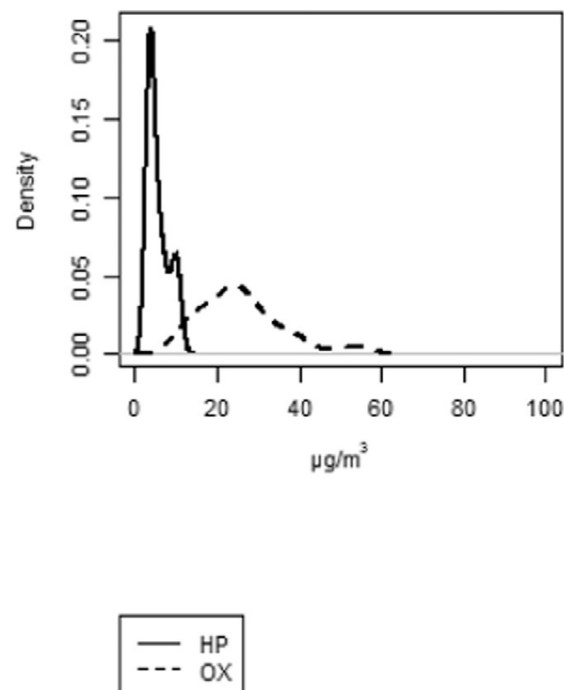

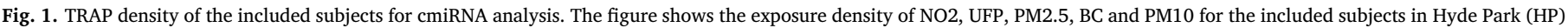
and Oxford Street (OX).

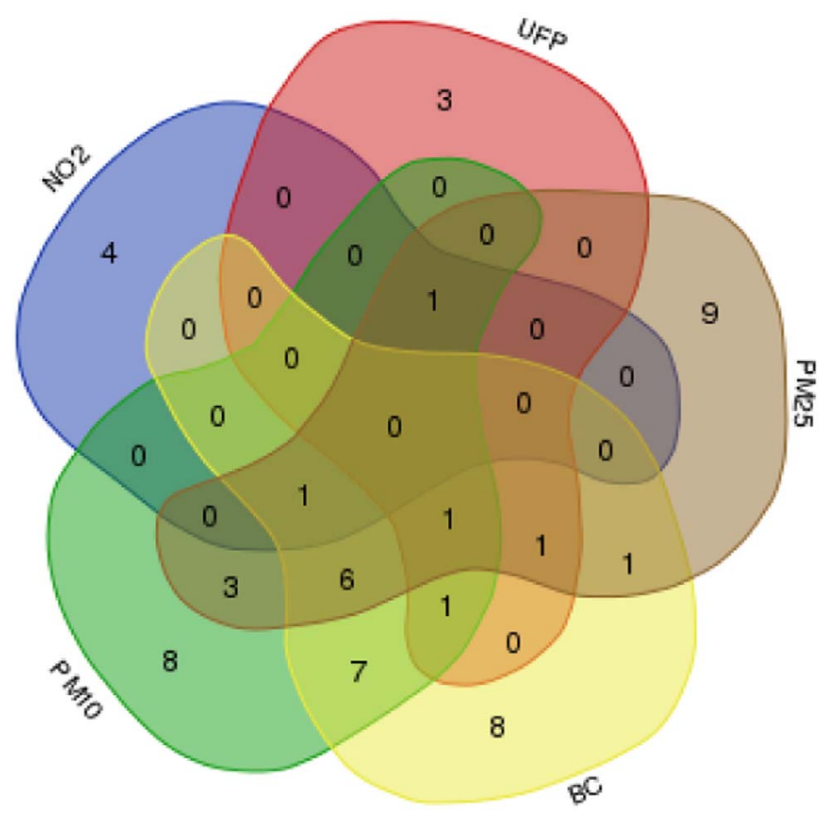

Fig. 2. Pollutant-specific cmiRNAs associated with TRAP exposure. The figure shows the overlap as well as the specificity of the pollutant-specific cmiRNAs associated with exposure to NO2, UFP, PM2.5, BC and PM10 of the included subjects in Hyde Park and Oxford Street.

\section{$-2.9 \log \mathrm{CPM}$ (Fig. 4).}

\subsection{Disease associations, target genes and pathway analysis}

To investigate the potential involvement of the 54 identified cmiRNAs in air pollutant-related diseases, we explored known diseasemiRNA interactions from published studies found in PubMed and miRWalk (version 2.0). We identified 8 interactions with breast cancer, 3 interactions with cardiovascular diseases, 5 interactions with inflammation, 8 interactions with respiratory disease (incl. lung cancer and Asthma), 6 interactions with neurodegenerative diseases (incl. Alzheimer's Disease and Parkinson) and 4 interaction with kidney diseases (Table 2).

To further describe the functional relevance of air pollutant exposure-associated cmiRNAs, we explored their potential interactions with gene targets from the experimentally validated miRNA-target interactions database miRTarBase (release 6 , only strong interactions). We identified a set of 491 target genes for the air pollutant exposureassociated 54 cmiRNAs. We explored the relationship between these cmiRNAs and their respective target genes by performing an overrepresentation analysis of KEGG pathways using ConsensusPathDB (release 32). This analysis identified 31 KEGG pathways (Supplementary Table S3). Notably, the list of overrepresented pathways includes significantly modified cancer-related pathways such as microRNAs in cancer, specifically breast cancer, non-small cell lung cancer and small cell lung cancer as well as signaling pathways such as the PI3K-Akt signaling pathway and the p53 signaling pathway.

\section{Discussion}

In this study, we evaluated the global circulating miRNome in 

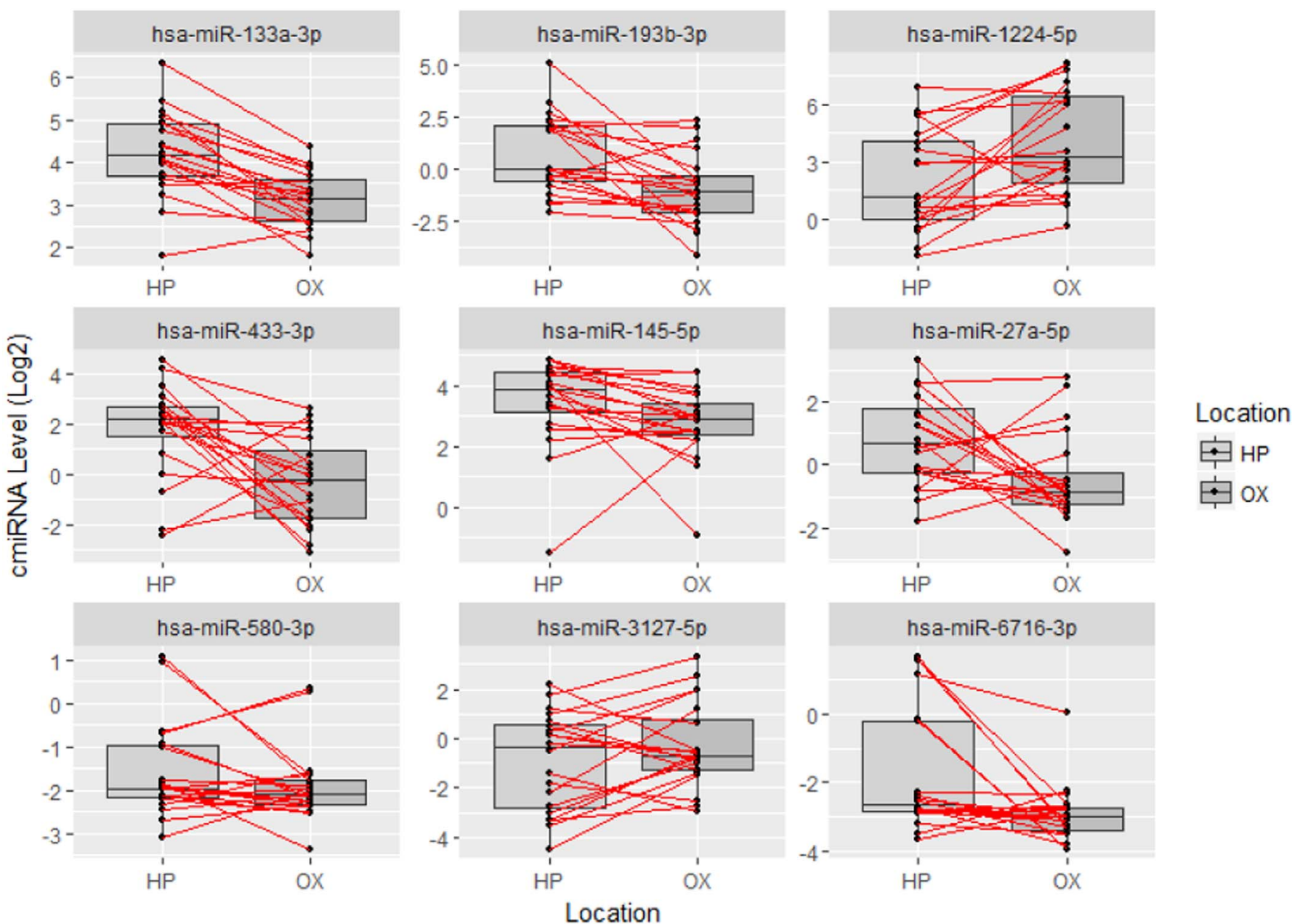

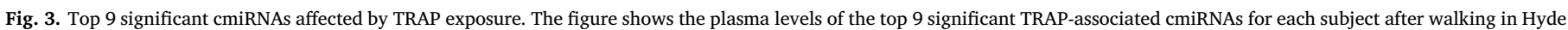

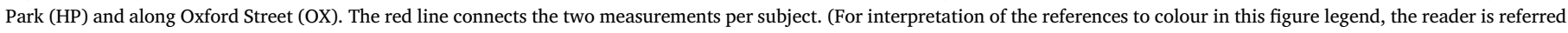
to the web version of this article.)

plasma from human subjects exposed to ambient TRAP for only $2 \mathrm{~h}$ by using NGS. We identified $54 \mathrm{cmiRNAs}$ that appear to be involved in the molecular response to NO2, UFP, PM2.5, BC and PM10 exposure. Next, we gathered information on tissue-specific miRNAs from those organs known to be targeted by ambient air pollutants. We found that the most abundant cmiRNAs present in plasma are equally expressed in all organs known to be targeted by TRAP. By contrast, the cmiRNAs present at low plasma levels appeared to be specifically enriched in certain target organs (TSI $>0.5$ ) (Fig. 4). The inverse association between high TSI and lower plasma level (correlation coefficient $=-0.68$ ) thus suggests that lower expressed $(\log \mathrm{CPM}<5)$ cmiRNAs might particularly represent tissue-specific biomarkers. Therefore, we hypothesize that the low abundant cmiRNAs are promising markers for the evaluation of tissue and organ conditions reflected by non-invasive 'liquid biopsies'. In contrast to studies in which miRNAs may leak into the circulation upon high exposures to toxic compounds, such as a consequence of acetaminophen-induced cytotoxicity in the liver, (Krauskopf et al., 2017b) ambient TRAP levels are unlikely to induce cell death within $2 \mathrm{~h}$ of time, implying that the induced alterations of cmiRNAs are more likely to reflect the active release of specific miRNAs into the circulation (Bollati et al., 2015).

Ambient TRAP exposure has been implicated in the development of cardiovascular disease, (Brook et al., 2010) respiratory disease, (Xing et al., 2016) kidney disease, (Bowe et al., 2017) several types of cancer, (Hamra et al., 2014; Tagliabue et al., 2016) as well as in neurodegenerative diseases (Cacciottolo et al., 2017; Chen et al., 2017). This is now also suggested on the molecular level by our results on tissue- and disease-specific cmiRNAs following short-term exposure to ambient TRAP. We demonstrated that the heart-enriched miR-133a-3p was significantly decreased in the plasma of $95 \%$ of the participants upon increased exposure. Downregulation or deletion of this miRNA has been associated with cardiac hypertrophy, severe fibrosis and heart failure. A study on the partial knockdown of miR-133 in mice has shown to induce hypertrophy during cardiac diseases (Care et al., 2007). Furthermore, the complete knockout of both miR-133 genes resulted in late embryonic or neonatal lethality due to ventricular septal defects in the majority of the mice, whereas mice that survived to adulthood developed extensive myocardial fibrosis and finally died from heart failure (Liu et al., 2008).

The smooth muscle cell-enriched miR-145-5p was significantly decreased in $80 \%$ of the participants upon exposure to TRAP. This miRNA, which has been reported to be downregulated in the carotid artery upon mechanical injury, is involved in regulation of smooth muscle contractility and the stress response to vessel injury, and thus is involved in cardiovascular diseases (Small et al., 2010).

Furthermore, we observed TRAP-dependent downregulation of the heart-enriched miR-499a-5p, a miRNA that is known to be downregulated under pathological heart conditions and has been suggested as a pharmacological target in the treatment of apoptosis-related cardiac disease, including myocardial infarction (Wang et al., 2011).

Overall, the two heart-enriched miRNAs miR-133a-3p and miR$499 a-5 p$ as well as the smooth muscle cell-enriched miR-145-5p play a 

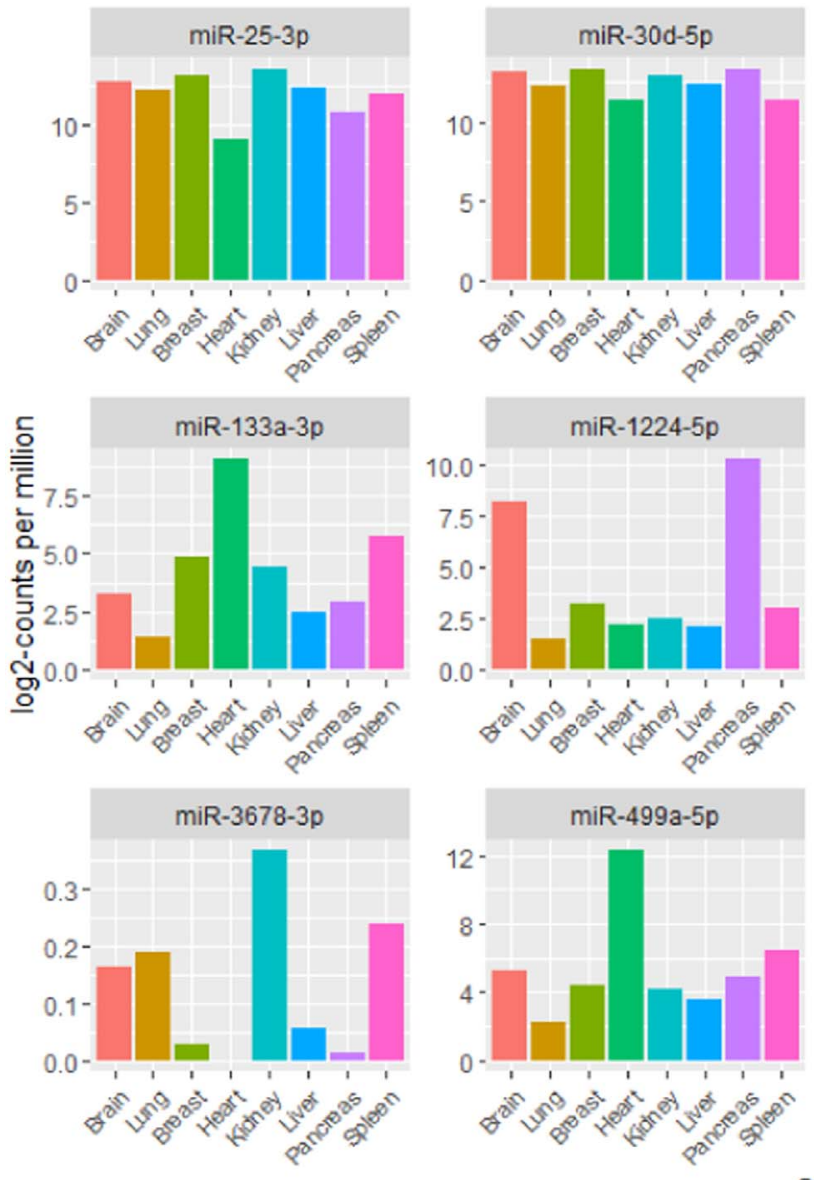
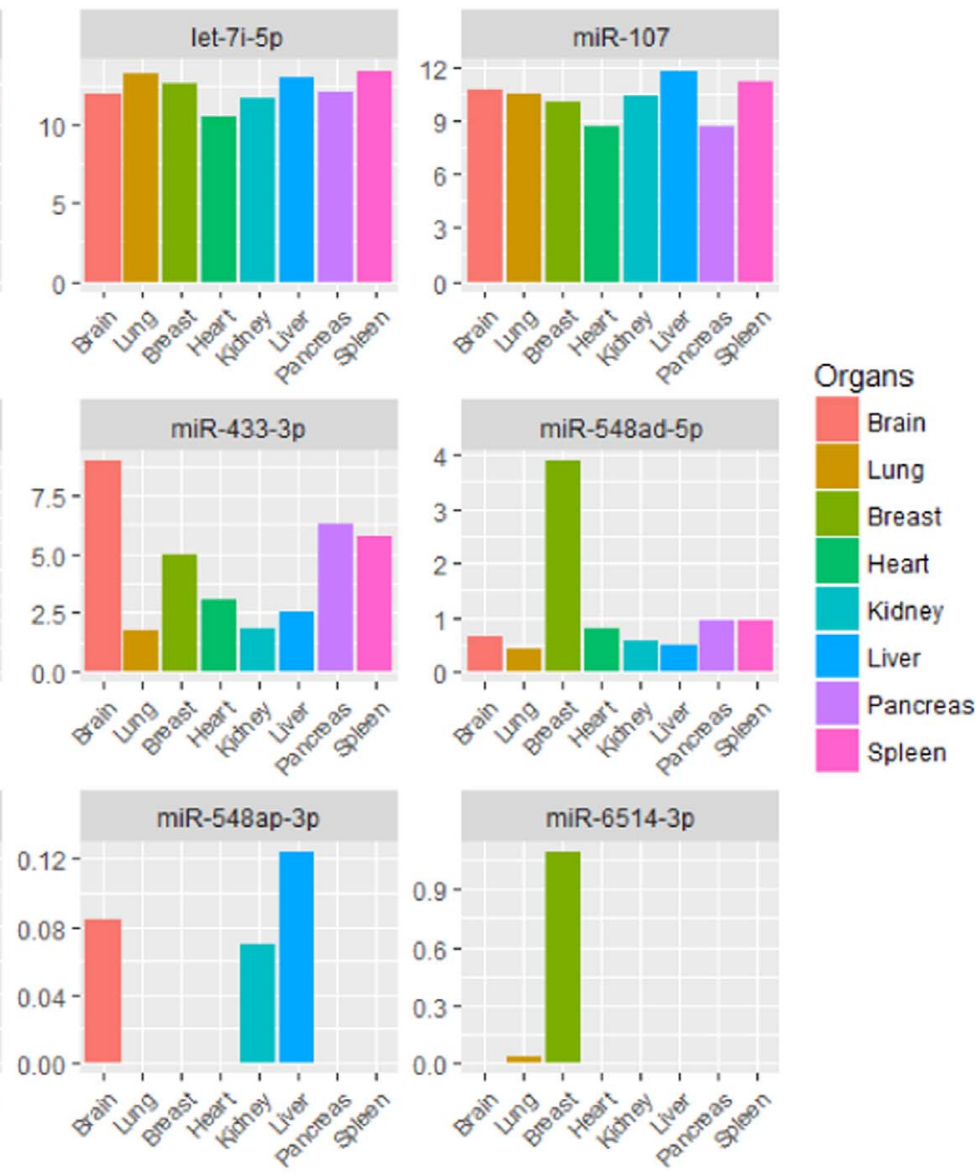

Organ

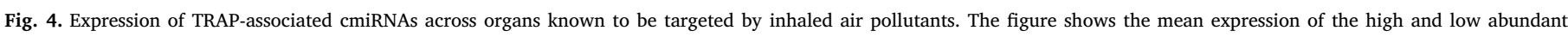

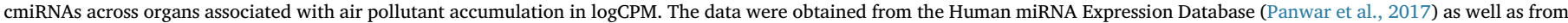

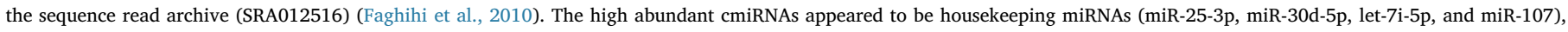
while the low abundant cmiRNAs seemed to show organ specificity/enrichment.

crucial role in the development of cardiovascular diseases which is known to be associated with exposure to TRAP, and therefore add potential value to a miRNA-based biomarker catalogue for cardiovascular disease prevention and biomarker-based environmental health risk assessment.

We observed, in $85 \%$ of the subjects decreased plasma levels of miR193b-3p in relation to higher TRAP exposure levels. The downregulation of this miRNA in breast tissue is known to enhance tumor progression and invasion in human breast cancer (Li et al., 2009). Also, the plasma level of miR-145-5p was decreased in $80 \%$ of participants, a miRNA that inhibits growth and migration of breast cancer (Zheng et al., 2016). Furthermore, miR-145-5p has also been identified to inhibit the proliferation of non-small cell lung cancer cells by targeting the oncogene $c-M y c$, and increasing the expression of miR-145 has been suggested as a novel approach for the treatment of lung cancer (Chen et al., 2010).

Table 2

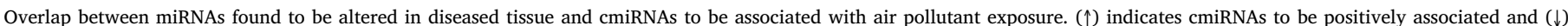

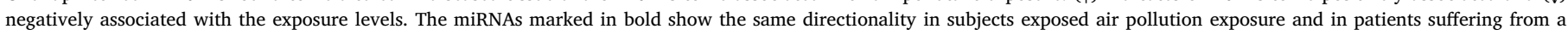
particular disease.

\begin{tabular}{|c|c|c|c|}
\hline Disease & $\begin{array}{l}\text { \# Associations/ } \\
\text { interactions }\end{array}$ & miRNAs & References \\
\hline Breast cancer & 8 & $\begin{array}{l}\operatorname{miR}-107 \uparrow ; \operatorname{miR}-125 a-5 p \downarrow ; \text { miR-145-5p } \downarrow ; \\
\text { miR-150-5p } \downarrow ; \text { miR-193b-3p } \downarrow ; \text { miR-342-3p } \downarrow \\
\text {; miR-34a-5p } \downarrow ; \text { miR-98-5p } \uparrow\end{array}$ & $\begin{array}{l}\text { (Martello et al., 2010; Nishida et al., 2011; Chen et al., 2010; } \\
\text { Lin et al., 2008; Li et al., 2009; Cittelly et al., 2010; Javeri } \\
\text { et al., 2013; Bhat-Nakshatri et al., 2009) }\end{array}$ \\
\hline Cardiovascular disease & 3 & miR-133a $\downarrow ;$ miR-145-5p $\downarrow ;$ miR-499a-5p $\downarrow$ & (Ito et al., 2010; Small et al., 2010; Wang et al., 2011) \\
\hline Inflammation & 5 & 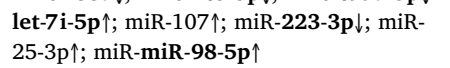 & $\begin{array}{l}\text { (Kumar et al., 2011; Schaefer et al., 2011; Wang et al., 2008a; } \\
\text { Kuhn et al., 2010) }\end{array}$ \\
\hline Kidney diseases & 4 & $\begin{array}{l}\operatorname{miR}-25-3 p \uparrow ; \operatorname{miR}-148 a-3 p \uparrow ; \operatorname{miR}-150-5 p \downarrow \\
\text { miR-223-3p } \downarrow\end{array}$ & 56 \\
\hline $\begin{array}{l}\text { Neurodegenerative diseases (incl. } \\
\text { Alzheimer's Disease and Parkinson) }\end{array}$ & 6 & $\begin{array}{l}\operatorname{miR}-107 \uparrow ; \text { miR-1224-5p } \uparrow ; \operatorname{miR}-145-5 p \downarrow \\
\text { miR-34a-5p } \downarrow ; \text { miR-433-3p } \downarrow ; \text { miR-885-5p } \downarrow\end{array}$ & $\begin{array}{l}\text { (Wang et al., 2008a; Wang et al., 2008b; Hoss et al., 2016; Tan } \\
\text { et al., 2014; Hooper et al., 2007; Burgos et al., 2014) }\end{array}$ \\
\hline $\begin{array}{l}\text { Respiratory diseases (incl. lung cancer } \\
\text { and asthma) }\end{array}$ & 8 & $\begin{array}{l}\text { let-7i-5p } \uparrow ; \text { miR-107 } \uparrow ; \text { miR-125a-5p } \downarrow ; \text { miR- } \\
\text { 145-5p } \downarrow ; \text { miR-148a-3p } \uparrow ; \text { miR-223-3p } \downarrow ; \\
\text { miR-574-5p } \uparrow \text { miR-98-5p } \uparrow\end{array}$ & $\begin{array}{l}\text { (Chen et al., 2010; Kumar et al., 2011; Garbacki et al., 2011; } \\
\text { Takahashi et al., 2009) }\end{array}$ \\
\hline
\end{tabular}


Potentially, the TRAP-induced inhibitions of miR-193b-3p and miR145-5p play a role in the complex progress of cancer development upon ambient air pollution exposure. Furthermore, in association with air pollutant exposure levels we identified another 4 decreased cmiRNAs (miR-125a-5, miR-150-5p, miR-342-3p and miR-34a-5p) and 2 increased cmiRNAs (miR-107 and miR-98-5p) for which a role in breast and/or lung cancer development has been described (Martello et al., 2010; Nishida et al., 2011; Chen et al., 2010; Lin et al., 2008; Li et al., 2009; Cittelly et al., 2010; Javeri et al., 2013; Bhat-Nakshatri et al., 2009).

The upregulation of miRNAs let-7i-5p and miR-98-5p has been shown to decrease IL-13 levels which resulted in the resolution of airway inflammation (Kumar et al., 2011). In this study we identified both miRNAs to be increased in plasma upon higher TRAP levels, thus potentially indicating a protective mechanism against increasing inflammation in the airways and circulation (McCreanor et al., 2007). We also identified the asthma-associated miRNAs miR-223-3p with decreased and miR-574-5p with increased plasma levels upon higher TRAP exposure. In lung tissue of mice challenged with ovalbumin mimicking acute, intermediate and chronic asthma, the downregulation of miR-223-3p to short term, and the upregulation of miR-574 to long term exposure to allergens has been reported (Garbacki et al., 2011).

Recently, TRAP exposure has also been linked to an increased risk of neurodegenerative diseases such as Alzheimer's and Parkinson's disease (Chen et al., 2017). We observed significantly decreased plasma levels of the brain-enriched miR-433-3p (85\% of all subjects) after exposure. This miRNA has also been found to be significantly decreased in the serum and cerebrospinal fluid of Parkinson's and Alzheimer's diseases patients when compared to healthy individuals (Burgos et al., 2014). The downregulation of cmiRNAs is unlikely to reflect cell damage as a consequence of toxic exposure, but rather suggests a biological response by a signaling molecule to a change in ambient air pollutant exposure intensity.

Plasma levels of another brain-enriched miRNA, miR-1224-5p, appeared increased in $80 \%$ of the study participants. This miRNA has been experimentally shown to silence LRRK2, which is known to be downregulated during pathogenesis of Parkinson's disease (Sibley et al., 2012; Li et al., 2014).

In patients diagnosed with Alzheimer's Disease, decreased serum levels of circulating miR-885 have been described (Tan et al., 2014). Also in our study we have observed decreased plasma levels of this miRNA in association with TRAP exposure. Furthermore, miR-107 and miR-34a-5p, both highly abundant in brain tissue, were found to be decreased in serum even in patients at the earliest stages of Alzheimer's Disease (Wang et al., 2008a; Hooper et al., 2007). While we observed increased miR-107 plasma levels with increased exposure intensity, miR-34a-5p levels were indeed decreased upon exposure.

Furthermore, we identified 4 TRAP-associated miRNAs, namely miR-25-3p, miR-148a-3p, miR-150-5 and miR-223-3p, which are known to be implicated in the pathogenesis and progression of kidney diseases (Wei et al., 2013). The miRNA which we identified most abundantly expressed in kidney, miR-148a-3p, was increased in plasma following higher TRAP levels. A closely related family member of this miRNA, miR-148b-3p, was found upregulated in peripheral blood mononuclear cells in patients suffering from igA nephropathy (Serino et al., 2012). More recently, serum levels of miR-148b-3p have been described as a potential non-invasive biomarker for diagnosing igA nephropathy (Serino et al., 2016). Interestingly, another study described low levels of miR-223-3p in circulating endothelial cells as a noninvasive method for evaluating the severity of igA nephropathy (Bao et al., 2014). In our analysis we found lower levels of miR-223-3p with increased TRAP levels. Our tissue specificity analysis revealed that miR-148a-3p and miR-223 are not enriched in kidney and were found abundantly expressed in other tissues (TSI $<0.37$ ). However, we identified another 6 kidney-enriched miRNAs $($ TSI $>0.68)$ that have not been sufficiently described in literature and might play a role in environmentally induced kidney diseases.

We evaluated the potential biological function of the cmiRNA signature by determining target genes, pathways and disease associations. The analysis showed that relevant disease pathways such as microRNAs in cancer, breast cancer, non-small cell lung cancer and small cell lung cancer as well as signaling pathways such as the PI3K-Akt signaling pathway and the p53 signaling pathway are potentially perturbed by exposure to ambient air pollution.

An earlier study reported 2 vesicle-associated cmiRNAs to be increased upon short-term exposure to metal-rich PM (miR-128 and miR302) in healthy workers (Bollati et al., 2015). In our analysis we observed decreased miR-128 levels but no change in miR-302 levels after short-term exposure. Another study on extracellular vesicle-bound cmiRNAs reported levels of 5 miRNAs to be changed after 6 months and 1 year in relation to ambient PM2.5 levels. Further 1 significantly decreased miRNA (miR-30d-5p) was reported after 1 day of exposure (Rodosthenous et al., 2016). This cmiRNA has also been identified in our analysis with significant decreased plasma levels already after $2 \mathrm{~h}$ of exposure to TRAP.

In this study we applied NGS to capture the complete plasma circulating miRNome and therefore were able to detect cmiRNAs that have not been investigated in relation to TRAP exposure previously. Despite the relatively small sample size, the experimental cross-over design of the study combined with the personal exposure monitoring enabled us to find significant associations of cmiRNAs and TRAP in the selected population.

The limited sample size, however, did not allow a meaningful analysis after stratification by disease state (COPD or IHD vs healthy) or gender. A hierarchical clustering analysis confirmed that cmiRNA expression is not confounded by disease status (data not shown). As a consequence of the study design in which COPD and IHD subjects were matched to healthy controls of the same age our analysis is limited to subjects of a mean age of 65.1 years (SD 7.7). Therefore the results need to be validated in other age groups to evaluate to which degree the results are transferable to other age groups. Due to the limited organ coverage of the human miRNA tissue atlas our analysis of miRNA tissue origin has been based on publically available sequencing data from various laboratories and therefore only suggests a potential origin of the detected cmiRNA. In summary, by applying stringent statistical analyses we have shown that the circulating miRNome is altered already after $2 \mathrm{~h}$ of exposure to ambient air pollution in a dose- and pollutant species-dependent manner. The cmiRNA signature seems to be capable to identify molecular mechanisms that upon perturbation by TRAP exposure are possibly involved in the complex pathogenesis of multiple diseases. Strikingly, the miRNA profile identified already after $2 \mathrm{~h}$ of TRAP exposure, presents specific associations with all major disease risks reported in relation to exposure to ambient air pollution. Next to the known disease risks that are affected by TRAP exposure our data also suggests aberrant abundance of circulating miRNAs that potentially have originated from the liver, pancreas and spleen. Since in addition, animal studies have demonstrated that in particular liver and spleen also present target organs for TRAP we suggest that future epidemiological studies should consider these organs to unravel their potential role in TRAP-associated pathology. Overall, our findings will not only contribute to the understanding of the complex coherence of environmental exposure and miRNAs but also provide insights into the pathomolecular process of TRAP-induced diseases, and thereby substantiate to biomarker-based health risk assessment. Consequently, we propose that a cmiRNA signature comprised of organ-enriched miRNAs presents a highly specific candidate for biomarker-based health risk assessment allowing the early detection and prevention of TRAP-induced health outcomes.

\section{Author contributions}

J.K., T.M.K. and J.C.K. designed the research. K.F.C., P. Cu., P. Co., 
B.B., F.J.K. and P.V. organized the epidemiologic part of the work. J.K. and R.S. performed the experiments. J.K., F.C., K.V., M.C. and R.V. analyzed the data. T.M.K. and J.C.K. supervised the project. J.K., T.M.K. and J.C.K. co-wrote and all authors commented on the paper.

\section{Additional information}

The authors declare no competing financial interests.

\section{Acknowledgments}

This work has been supported by the European Union within the frame of the Exposomics (226756) project and the British Heart Foundation (PGF/10/82/28608).

\section{Appendix A. Supplementary data}

Supplementary data to this article can be found online at https:// doi.org/10.1016/j.envint.2018.01.014.

\section{References}

Bao, H., et al., 2014. MiR-223 downregulation promotes glomerular endothelial cell activation by upregulating importin alpha4 and alpha5 in IgA nephropathy. Kidney Int. 85, 624-635. http://dx.doi.org/10.1038/ki.2013.469.

Baras, A.S., et al., 2015. miRge - A Multiplexed Method of Processing Small RNA-Seq Data to Determine MicroRNA Entropy. PLoS One 10, e0143066. http://dx.doi.org/10. 1371/journal.pone.0143066.

Bartel, D.P., 2009. MicroRNAs: target recognition and regulatory functions. Cell 136, 215-233. http://dx.doi.org/10.1016/j.cell.2009.01.002.

Bates, D., Machler, M., Bolker, B.M., Walker, S.C., 2015. Fitting linear mixed-effects models using lme4. J. Stat. Softw. 67, 1-48.

Benjamini, Y., Hochberg, Y., 1995. Controlling the false discovery rate - a practical and powerful approach to multiple testing. J. Roy. Stat. Soc. B Met. 57, 289-300.

Bhat-Nakshatri, P., et al., 2009. Estradiol-regulated microRNAs control estradiol response in breast cancer cells. Nucleic Acids Res. 37, 4850-4861. http://dx.doi.org/10.1093/ nar/gkp500.

Block, M.L., Calderon-Garciduenas, L., 2009. Air pollution: mechanisms of neuroinflammation and CNS disease. Trends Neurosci. 32, 506-516. http://dx.doi.org/10. 1016/j.tins.2009.05.009.

Bollati, V., et al., 2015. Microvesicle-associated microRNA expression is altered upon particulate matter exposure in healthy workers and in A549 cells. J. Appl. Toxicol. 35, 59-67. http://dx.doi.org/10.1002/jat.2987.

Bowe, B., et al., 2017. Particulate matter air pollution and the risk of incident CKD and progression to ESRD. J. Am. Soc. Nephrol. http://dx.doi.org/10.1681/ASN. 2017030253.

Brook, R.D., et al., 2010. Particulate matter air pollution and cardiovascular disease: an update to the scientific statement from the American Heart Association. Circulation 121, 2331-2378. http://dx.doi.org/10.1161/CIR.0b013e3181dbece1.

Burgos, K., et al., 2014. Profiles of extracellular miRNA in cerebrospinal fluid and serum from patients with Alzheimer's and Parkinson's diseases correlate with disease status and features of pathology. PLoS One 9, e94839. http://dx.doi.org/10.1371/journal. pone.0094839.

Cacciottolo, M., et al., 2017. Particulate air pollutants, APOE alleles and their contributions to cognitive impairment in older women and to amyloidogenesis in experimental models. Transl. Psychiatry 7, e1022. http://dx.doi.org/10.1038/tp.2016.280.

Care, A., et al., 2007. MicroRNA-133 controls cardiac hypertrophy. Nat. Med. 13, 613-618. http://dx.doi.org/10.1038/nm1582.

Chen, Z., et al., 2010. miRNA-145 inhibits non-small cell lung cancer cell proliferation by targeting c-Myc. J. Exp. Clin. Cancer Res. 29, 151. http://dx.doi.org/10.1186/17569966-29-151.

Chen, H., et al., 2017. Living near major roads and the incidence of dementia, Parkinson's disease, and multiple sclerosis: a population-based cohort study. Lancet 389 , 718-726. http://dx.doi.org/10.1016/S0140-6736(16)32399-6.

Cittelly, D.M., et al., 2010. Downregulation of miR-342 is associated with tamoxifen resistant breast tumors. Mol. Cancer 9, 317. http://dx.doi.org/10.1186/1476-4598-9. 317.

Faghihi, M.A., et al., 2010. Evidence for natural antisense transcript-mediated inhibition of microRNA function. Genome Biol. 11, R56. http://dx.doi.org/10.1186/gb-201011-5-r56.

Falcon-Rodriguez, C.I., Osornio-Vargas, A.R., Sada-Ovalle, I., Segura-Medina, P., 2016. Aeroparticles, Composition, and Lung Diseases. Front. Immunol. 7, 3. http://dx.doi org /10.3389/fimmu.2016.00003.

Garbacki, N., et al., 2011. MicroRNAs Profiling in murine models of acute and chronic asthma: a relationship with mRNAs targets. PLoS One 6, e16509. http://dx.doi.org 10.1371/journal.pone.0016509.

Georgiadis, P., et al., 2016. Omics for prediction of environmental health effects: blood leukocyte-based cross-omic profiling reliably predicts diseases associated with tobacco smoking. Sci. Rep. 6, 20544. http://dx.doi.org/10.1038/srep20544.
Griffiths-Jones, S., Grocock, R.J., van Dongen, S., Bateman, A., Enright, A.J., 2006. miRBase: microRNA sequences, targets and gene nomenclature. Nucleic Acids Res. 34, D140-144. http://dx.doi.org/10.1093/nar/gkj112.

Hamra, G.B., et al., 2014. Outdoor particulate matter exposure and lung cancer: a systematic review and meta-analysis. Environ. Health Perspect. 122, 906-911. http:// dx.doi.org/10.1289/ehp.1408092.

Hooper, C., et al., 2007. p53 is upregulated in Alzheimer's disease and induces tau phosphorylation in HEK293a cells. Neurosci. Lett. 418, 34-37. http://dx.doi.org/10. 1016/j.neulet.2007.03.026.

Hoss, A.G., Labadorf, A., Beach, T.G., Latourelle, J.C., Myers, R.H., 2016. microRNA profiles in Parkinson's disease prefrontal cortex. Front. Aging Neurosci. 8, 36. http:// dx.doi.org/10.3389/fnagi.2016.00036.

Hunter, M.P., et al., 2008. Detection of microRNA expression in human peripheral blood microvesicles. PLoS One 3, e3694. http://dx.doi.org/10.1371/journal.pone. 0003694.

Intarasunanont, P., et al., 2012. Effects of arsenic exposure on DNA methylation in cord blood samples from newborn babies and in a human lymphoblast cell line. Environ. Health 11, 31. http://dx.doi.org/10.1186/1476-069X-11-31.

Ito, T., Yagi, S., Yamakuchi, M., 2010. MicroRNA-34a regulation of endothelial senescence. Biochem. Biophys. Res. Commun. 398, 735-740. http://dx.doi.org/10.1016/j. bbrc.2010.07.012.

Javeri, A., Ghaffarpour, M., Taha, M.F., Houshmand, M., 2013. Downregulation of miR$34 \mathrm{a}$ in breast tumors is not associated with either p53 mutations or promoter hypermethylation while it correlates with metastasis. Med. Oncol. 30, 413. http://dx. doi.org/10.1007/s12032-012-0413-7.

Kirschner, M.B., et al., 2013. The impact of hemolysis on cell-free microRNA biomarkers. Front. Genet. 4, 94. http://dx.doi.org/10.3389/fgene.2013.00094.

Krauskopf, J., Verheijen, M., Kleinjans, J.C., de Kok, T.M., Caiment, F., 2015. Development and regulatory application of microRNA biomarkers. Biomark. Med 9, 1137-1151. http://dx.doi.org/10.2217/bmm.15.50.

Krauskopf, J., et al., 2017a. MicroRNA profile for health risk assessment: environmental exposure to persistent organic pollutants strongly affects the human blood microRNA machinery. Sci. Rep. 7, 9262. http://dx.doi.org/10.1038/s41598-017-10167-7.

Krauskopf, J., et al., 2017b. Serum microRNA signatures as "liquid biopsies" for interrogating hepatotoxic mechanisms and liver pathogenesis in human. PLoS One 12, e0177928. http://dx.doi.org/10.1371/journal.pone.0177928.

Kroh, E.M., Parkin, R.K., Mitchell, P.S., Tewari, M., 2010. Analysis of circulating microRNA biomarkers in plasma and serum using quantitative reverse transcriptionPCR (qRT-PCR) (vol 50, pg 298, 2010). Methods 52, 268.

Kuhn, A.R., et al., 2010. MicroRNA expression in human airway smooth muscle cells: role of miR-25 in regulation of airway smooth muscle phenotype. Am. J. Respir. Cell Mol. Biol. 42, 506-513. http://dx.doi.org/10.1165/rcmb.2009-01230C.

Kumar, M., et al., 2011. Let-7 microRNA-mediated regulation of IL-13 and allergic airway inflammation. J. Allergy Clin. Immunol. 128 (1077-1085), e1071-1010. http://dx. doi.org/10.1016/j.jaci.2011.04.034.

Landgraf, P., et al., 2007. A mammalian microRNA expression atlas based on small RNA library sequencing. Cell 129, 1401-1414. http://dx.doi.org/10.1016/j.cell.2007.04. 040.

Lee, B.J., Kim, B., Lee, K., 2014. Air pollution exposure and cardiovascular disease. Toxicol. Res. 30, 71-75. http://dx.doi.org/10.5487/TR.2014.30.2.071.

Li, X.F., Yan, P.J., Shao, Z.M., 2009. Downregulation of miR-193b contributes to enhance urokinase-type plasminogen activator (UPA) expression and tumor progression and invasion in human breast cancer. Oncogene 28, 3937-3948. http://dx.doi.org/10. 1038/onc.2009.245.

Li, J.Q., Tan, L., Yu, J.T., 2014. The role of the LRRK2 gene in Parkinsonism. Mol. Neurodegener. 9, 47. http://dx.doi.org/10.1186/1750-1326-9-47.

$\mathrm{Li}, \mathrm{Q}$., et al., 2015. The preferential accumulation of heavy metals in different tissues following frequent respiratory exposure to PM2.5 in rats. Sci. Rep. 5, 16936. http:// dx.doi.org/10.1038/srep16936.

Lin, Y.C., et al., 2008. c-Myb is an evolutionary conserved miR-150 target and miR-150/cMyb interaction is important for embryonic development. Mol. Biol. Evol. 25 2189-2198. http://dx.doi.org/10.1093/molbev/msn165.

Liu, N., et al., 2008. microRNA-133a regulates cardiomyocyte proliferation and suppresses smooth muscle gene expression in the heart. Genes Dev. 22, 3242-3254. http://dx.doi.org/10.1101/gad.1738708.

Martello, G., et al., 2010. A microRNA targeting dicer for metastasis control. Cell 141, 1195-1207. http://dx.doi.org/10.1016/j.cell.2010.05.017.

McCreanor, J., et al., 2007. Respiratory effects of exposure to diesel traffic in persons with asthma. N. Engl. J. Med. 357, 2348-2358. http://dx.doi.org/10.1056/ NEJMoa071535.

Nishida, N., et al., 2011. MicroRNA-125a-5p is an independent prognostic factor in gastric cancer and inhibits the proliferation of human gastric cancer cells in combination with Trastuzumab. Clin. Cancer Res. 17, 2725-2733. http://dx.doi.org/10.1158/ 1078-0432.Ccr-10-2132.

Oberdorster, G., et al., 2004. Translocation of inhaled ultrafine particles to the brain. Inhal. Toxicol. 16, 437-445, http://dx doi.org/10.1080/08958370490439597.

Panwar, B., Omenn, G.S., Guan, Y., 2017. miRmine: A database of human miRNA expression profiles. Bioinformatics. http://dx.doi.org/10.1093/bioinformatics/btx019.

Pinheiro, J., Bates, D., DebRoy, S., Sarkar, D., R Core Team, 2014. nlme: linear and nonlinear mixed effects models. R package version 3.1-117. Available at. http:// CRAN.R-project.org/package = nlmepp. 2014.

Ritchie, M.E., et al., 2015. limma powers differential expression analyses for RNA-sequencing and microarray studies. Nucleic Acids Res. 43, e47. http://dx.doi.org/10. 1093/nar/gkv007.

Rodosthenous, R.S., et al., 2016. Ambient particulate matter and microRNAs in extracellular vesicles: a pilot study of older individuals. Part. Fibre Toxicol 13, 13. http:// 
dx.doi.org/10.1186/s12989-016-0121-0.

Schaefer, J.S., Montufar-Solis, D., Vigneswaran, N., Klein, J.R., 2011. Selective upregulation of microRNA expression in peripheral blood leukocytes in IL-10 ${ }^{-/-}$mice precedes expression in the colon. J. Immunol. 187, 5834-5841. http://dx.doi.org/10. 4049/jimmunol.1100922.

Serino, G., Sallustio, F., Cox, S.N., Pesce, F., Schena, F.P., 2012. Abnormal miR-148b expression promotes aberrant glycosylation of IgA1 in IgA nephropathy. J. Am. Soc. Nephrol. 23, 814-824. http://dx.doi.org/10.1681/ASN.2011060567.

Serino, G., et al., 2016. In a retrospective international study, circulating miR-148b and let-7b were found to be serum markers for detecting primary IgA nephropathy. Kidney Int. 89, 683-692. http://dx.doi.org/10.1038/ki.2015.333.

Sibley, C.R., Seow, Y., Curtis, H., Weinberg, M.S., Wood, M.J.A., 2012. Silencing of Parkinson's disease-associated genes with artificial mirtron mimics of miR-1224 Nucleic Acids Res. 40, 9863-9875. http://dx.doi.org/10.1093/nar/gks712.

Sinharay, R., et al., 2017. Respiratory and cardiovascular responses to walking down a traffic-polluted road compared with walking in a traffic-free area in participants aged 60 years and older with chronic lung or heart disease and age-matched healthy controls: a randomised, crossover study. Lancet. http://dx.doi.org/10.1016/S01406736(17)32643-0.

Small, E.M., Frost, R.J., Olson, E.N., 2010. MicroRNAs add a new dimension to cardiovascular disease. Circulation 121, 1022-1032. http://dx.doi.org/10.1161/ CIRCULATIONAHA.109.889048.

Tagliabue, G., et al., 2016. Atmospheric fine particulate matter and breast cancer mortality: a population-based cohort study. BMJ Open 6, e012580. http://dx.doi.org/10. 1136/bmjopen-2016-012580.

Takahashi, Y., et al., 2009. MiR-107 and MiR-185 can induce cell cycle arrest in human non small cell lung cancer cell lines. PLoS One 4, e6677. http://dx.doi.org/10.1371/ journal.pone.0006677.

Tan, L., et al., 2014. Genome-wide serum microRNA expression profiling identifies serum biomarkers for Alzheimer's disease. J. Alzheimers Dis. 40, 1017-1027. http://dx.doi. org/10.3233/JAD-132144.

Turchinovich, A., Weiz, L., Burwinkel, B., 2012. Extracellular miRNAs: the mystery of their origin and function. Trends Biochem. Sci. 37, 460-465. http://dx.doi.org/10 1016/j.tibs.2012.08.003. van Leeuwen, D.M., et al., 2006. Genome-wide differential gene expression in children exposed to air pollution in the Czech Republic. Mutat. Res. 600, 12-22. http://dx.doi. org/10.1016/j.mrfmmm.2006.05.032.

Vriens, A., et al., 2016. Recent exposure to ultrafine particles in school children alters miR-222 expression in the extracellular fraction of saliva. Environ. Health 15, 80 http://dx.doi.org/10.1186/s12940-016-0162-8.

Vrijens, K., Bollati, V., Nawrot, T.S., 2015. MicroRNAs as potential signatures of environmental exposure or effect: a systematic review. Environ. Health Perspect. 123, 399-411. http://dx.doi.org/10.1289/ehp.1408459.

Wang, W.X., et al., 2008a. The expression of microRNA miR-107 decreases early in Alzheimer's disease and may accelerate disease progression through regulation of beta-site amyloid precursor protein-cleaving enzyme 1. J. Neurosci. 28, 1213-1223. http://dx.doi.org/10.1523/JNEUROSCI.5065-07.2008.

Wang, G., et al., 2008b. Variation in the miRNA-433 binding site of FGF20 confers risk for Parkinson disease by overexpression of alpha-synuclein. Am. J. Hum. Genet. 82, 283-289. http://dx.doi.org/10.1016/j.ajhg.2007.09.021.

Wang, J.X., et al., 2011. miR-499 regulates mitochondrial dynamics by targeting calcineurin and dynamin-related protein-1. Nat. Med. 17, 71-78. http://dx.doi.org/10. 1038/nm.2282.

Wei, Q., Mi, Q.S., Dong, Z., 2013. The regulation and function of microRNAs in kidney diseases. IUBMB Life 65, 602-614. http://dx.doi.org/10.1002/iub.1174.

Xing, Y.F., Xu, Y.H., Shi, M.H., Lian, Y.X., 2016. The impact of PM2.5 on the human respiratory system. J. Thorac. Dis. 8, E69-74. http://dx.doi.org/10.3978/j.issn.20721439.2016.01.19.

Yaghjyan, L., et al., 2017. Association between air pollution and mammographic breast density in the breast cancer surveillance consortium. Breast Cancer Res. 19, 36. http://dx.doi.org/10.1186/s13058-017-0828-3.

Yanai, I., et al., 2005. Genome-wide midrange transcription profiles reveal expression level relationships in human tissue specification. Bioinformatics 21, 650-659. http:// dx.doi.org/10.1093/bioinformatics/bti042.

Zheng, M.Z., Sun, X., Li, Y.Q., Zuo, W.S., 2016. MicroRNA-145 inhibits growth and migration of breast cancer cells through targeting oncoprotein ROCK1. Tumor Biol. 37, 8189-8196. http://dx.doi.org/10.1007/s13277-015-4722-2. 